\title{
Miocárdio não compactado de ventrículo esquerdo: relato de caso com estudo de necropsia
}

\section{unitermos}

Cardiomiopatias

Miocárdio ventricular não compactado isolado

Necrópsia

\section{resumo}

O miocárdio não compactado de ventrículo esquerdo é cardiomiopatia genética em decorrência de uma anormalidade da morfogênese endomiocárdica. Quando presente, geralmente está associado a outras anomalias cardíacas congênitas, sendo raro seu achado isolado sem outras alterações estruturais cardíacas associadas. Desde seu primeiro relato em 1990, a não compactação do miocárdio vem sendo amplamente estudada a fim de se estabelecerem caráter genético, história natural, métodos de diagnóstico, repercussões hemodinâmicas e prognóstico. $\mathrm{O}$ objetivo deste estudo foi relatar um caso de miocárdio não compactado isolado de ventrículo esquerdo com estudo de necrópsia. abstract

Noncompaction of the ventricular myocardium is a genetic cardiomyopathy due to an alteration in endomyocardial morphogenesis. When present, it is commonly associated with other congenital cardiac diseases, whereas it is rare as an isolated finding without other associated cardiac structural alterations. Since its first report in 1990, the noncompaction of the ventricular myocardium has been widely studied in order to establish its genetic character, natural history, diagnostic methods, hemodynamic repercussions and prognosis. This study aims to report the case of an isolated noncompaction of the left ventricular myocardium with autopsy.

\section{key words}

Cardiomyopathies

Isolated noncompaction of ventricular myocardium

Autopsy

1. Faculdade de Medicina do Centro Ciências da Vida da Pontifíia Universidade Católica de Campinas (PUC-Campinas). 


\section{Introdução}

Em 2006 a American Heart Association (AHA) incluiu a não compactação do miocárdio ventricular na classificação das cardiomiopatias como cardiomiopatia genética ${ }^{(6)}$. Desde seu primeiro relato, em 1990, por Chin et al., o miocárdio não compactado isolado de ventrículo esquerdo vem sendo amplamente estudado a fim de se estabelecerem caráter genético, história natural, métodos de diagnóstico, repercussões hemodinâmicas e prognóstico(2).

Durante a fase inicial do desenvolvimento embrionário, o coração consiste em uma trama desconexa de fibras musculares, separadas por profundos recessos chamados espaços intertrabeculares (ou sinusoides) que se comunicam com as câmaras do coração. À medida que o coração se desenvolve, o miocárdio se condensa gradualmente, e os recessos intertrabeculares são reduzidos a capilares ou desaparecem. A compactação das fibras é normalmente mais completa no ventrículo esquerdo, predominantemente na porção distal (apical), que no ventrículo direito(2).

Por conseguinte, o miocárdio ventricular não compactado é definido como uma cardiomiopatia genética em decorrência de uma anormalidade da morfogênese endomiocárdica que acredita tratar-se de uma parada da compactação das fibras miocárdicas que se encontram, formando uma rede frouxa de fibras musculares entrelaçadas ${ }^{(2,6)}$.

O miocárdio não compactado de ventrículo esquerdo pode ser um achado isolado ou estar associado a outras anomalias cardíacas congênitas, em especial as cardiopatias congênitas cianóticas (2). Com base em estudos ecocardiográficos, a prevalência do miocárdio não compactado isolado, definido pela ausência de outra alteração estrutural cardíaca associada, foi estimada em $0,05 \%$ na população geral, o que demonstra a raridade dessa entidade ${ }^{(3,8)}$.

O ecocardiograma transtorácico constitui excelente método diagnóstico na avaliação não invasiva da estrutura anatômica e funcional do ventrículo esquerdo ${ }^{(2,3,6,8,9)}$. O diagnóstico ecocardiográfico envolve a observação dos recessos intertrabeculares visualizados no ecocardriograma, como espaços no endocárdio; e ao Doppler, fluxo sanguíneo direcionado do espaço ventricular para esses recessos ${ }^{(7)}$. Ainda, outros métodos de aquisição de imagem foram estudados, como angiografia de ventrículo esquerdo ${ }^{(4,6)}$, ressonância nuclear magnética (RNM) cardía$\mathrm{Ca}^{(4,6,7)}$, tomografia computadorizada $(\mathrm{TC})^{(4)}$ e cintilografia com tálio-201 ${ }^{(4)}$.
A história natural da doença é indeterminada e diversa, o que facilita interpretações errôneas ${ }^{(6)}$. O quadro clínico pode apresentar-se como insuficiência cardíaca, disfunção sistólica do ventrículo esquerdo, tromboembolismo, arritmias, morte súbita e diversas formas de remodelamento ${ }^{(2,3,5-9)}$. Por ser muitas vezes um achado ecocardiográfico, a grande maioria dos pacientes apresenta-se assintomática, mas com perda progressiva e gradual da função ventricular esquerda, apresentando sinais de importante insuficiência cardíaca( ${ }^{(5)}$.

O objetivo deste estudo foi discutir essa entidade nosológica, de conhecimento médico recente, por meio do relato de caso de uma portadora de miocárdio não compactado diagnosticado em nosso serviço, descrevendo os achados de histórias clínica e familiar, estratificação diagnóstica e abordagem terapêutica, acompanhados de uma revisão literária, em que são descritos aspectos clínicos, diagnósticos, prognósticos e terapêuticos. Por intermédio da realização da necropsia, buscamos correlacionar todo o quadro clínico e confrontar as alterações estruturais encontradas com as descritas na literatura.

\section{Relato do caso}

Paciente do sexo feminino, 16 anos, parda, acompanhada com quadro de insuficiência cardíaca devido à cardiomiopatia dilatada. Há um dia foi internada com queixa de dor abdominal em epigastro e hipocôndrio esquerdo. Nos antecedentes: irmã mais velha falecida há um ano por morte súbita; consanguinidade dos pais. O exame físico evidenciou estase jugular a $45^{\circ}$, bulhas arrítmicas com bigeminismo e trigeminismo e sopro sistólico em borda esternal esquerda. O eletrocardiograma evidenciava sobrecarga atrial direita, alteração difusa da repolarização ventricular e retificação do segmento ST em parede lateral; radiografias de tórax mostraram aumento da área cardíaca, abaulamento do arco médio e parênquima pulmonar normotransparente; ecocardiograma transtorácico demonstrou refluxo mitral e tricúspide moderado, fração de ejeção calculada de $10 \%$, ausência de outras anormalidades cardíacas e proeminentes e numerosas trabéculas com recessos intertrabeculares profundos. A cintilografia miocárdica com gálio foi positiva $+2 /+4$ para miocardite; e a ultrassonografia abdominal mostrou colecistite aguda litiásica, ascite, derrame pleural bilateral e redução dos ruídos hidroaéreos. A paciente foi submetida à RNM, que evidenciou derrame pericárdico, hipocinesia 
difusa das paredes de ambos os ventrículos com déficit de função sistólica, insuficiência mitral $+2 /+4$, aumento das câmaras cardíacas, em especial do ventrículo esquerdo que apresentava suas paredes com dupla camada, sendo a epimiocárdica compactada e a endomiocárdica, de aspecto esponjoso-frouxo. De acordo com critérios clínicos e resultados dos exames complementares, diagnosticouse miocárdio não compactado de ventrículo esquerdo de forma isolada. Houve acentuada piora do quadro de dor abdominal, além de diminuição da diurese, hipotensão e crise convulsiva. Evoluiu com parada cardiorrespiratória não responsiva às manobras de reanimação.

\section{Discussão}

As cardiomiopatias são um grupo de doenças em que o envolvimento do próprio músculo cardíaco é a característica dominante da apresentação clínica, causando disfunção mecânica e/ou elétrica e cursando frequentemente hipertrofia ou dilatação ventricular e falência cardíaca ${ }^{(6)}$. Atualmente, as cardiomiopatias são classificadas pelas características patofisiológicas predominantes e, se possível, por atores etiológicos e patogênicos. Em 2006 a AHA incluiu a não compactação do miocárdio ventricular na classificação das cardiomiopatias como cardiomiopatia genética ${ }^{(6)}$. Desde seu primeiro relato, em 1990, por Chin et al., o miocárdio não compactado isolado de ventrículo esquerdo vem sendo amplamente estudado, a fim de se estabelecerem caráter genético, história natural, métodos de diagnóstico, repercussões hemodinâmicas e prognóstico(2).

O miocárdio ventricular não compactado é definido como uma cardiomiopatia genética, em decorrência de uma anormalidade da morfogênese endomiocárdica, que acredita tratar-se de uma parada da compactação das fibras miocárdicas que se encontram, formando uma rede frouxa de fibras musculares entrelaçadas ${ }^{(2,6)}$. Geralmente está associado a outras anomalias cardíacas congênitas, em especial as cardiopatias congênitas cianóticas, sendo raro seu achado isolado sem outras alterações estruturais cardíacas associadas, como neste caso ${ }^{(2)}$.

O estudo de necropsia revelou o coração aumentado de tamanho, refletindo hipertrofias ventriculares direita e esquerda evidentes. Embora os ventrículos sejam mais intensamente afetados do que os átrios, observou-se que todas as câmaras do coração estavam dilatadas. Ainda, chamamos a atenção para o fato de que a dilatação ven- tricular esquerda foi tão intensa, de modo que a parede do ventrículo esquerdo parece ter a espessura normal ou até mesmo mais fina. O miocárdio se apresentou esponjoso, não compactado, flácido e pálido, e cicatrizes subendocárdicas podem ser visualizadas, assim como a vasta presença dos recessos comunicantes com a câmara ventricular. As trabeculações eram menos proeminentes e menos numerosas próximas à válvula mitral com relação à ponta do ventrículo esquerdo. No caso analisado em nosso serviço, por meio da realização da necropsia, obtivemos resultados semelhantes aos encontrados na literatura disponível, com predomínio de trabeculações na região ântero-apical e sem a existência de outras anomalias. O endocárdio do ventrículo esquerdo, especialmente no ápice, que normalmente tende a ser espessado, encontra-se fino e caracteristicamente esponjoso. $E$, apesar de muitas vezes estarem presentes, não encontramos trombos murais aderidos nessa área. Havia moderada insuficiência mitral por disfunção papilar e tricúspide. As artérias coronárias estavam pérvias. A complicação que agravou o quadro foi a trombose da veia cava inferior no nível das veias renais.

Microscopicamente, o miocárdio não compactado caracterizou-se por uma rede de fibras musculares espa-

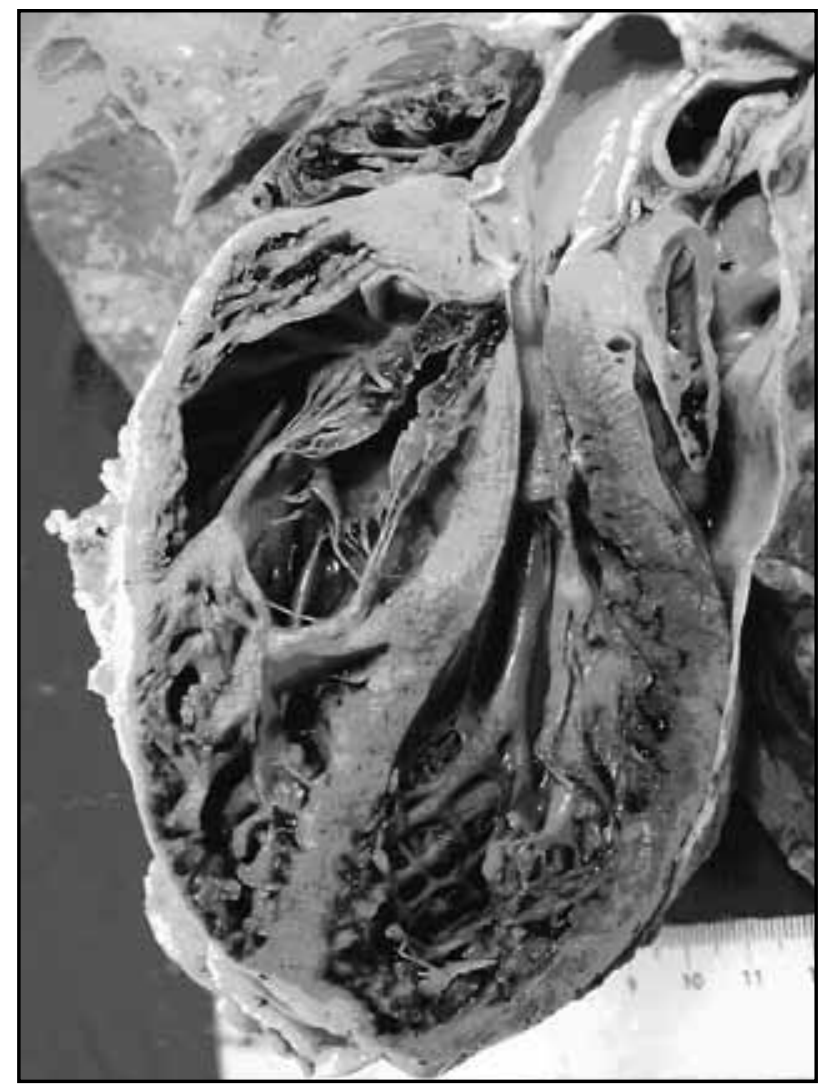

Figura 1 - Macroscopia do coração com miocárdio não compactado (esponjoso) nos terços médio e apical do ventrículo esquerdo 
çadas, atróficas e algumas hipertróficas. Os miócitos cardíacos, especialmente no subendocárdio, demonstraram alterações degenerativas avançadas, caracterizadas por perda de miofibrilas, um efeito que dá às células aparência vazia ou vacuolada. Fibrose intersticial e perivascular no miocárdio é evidente, sendo também mais proeminente na zona subendocárdica.

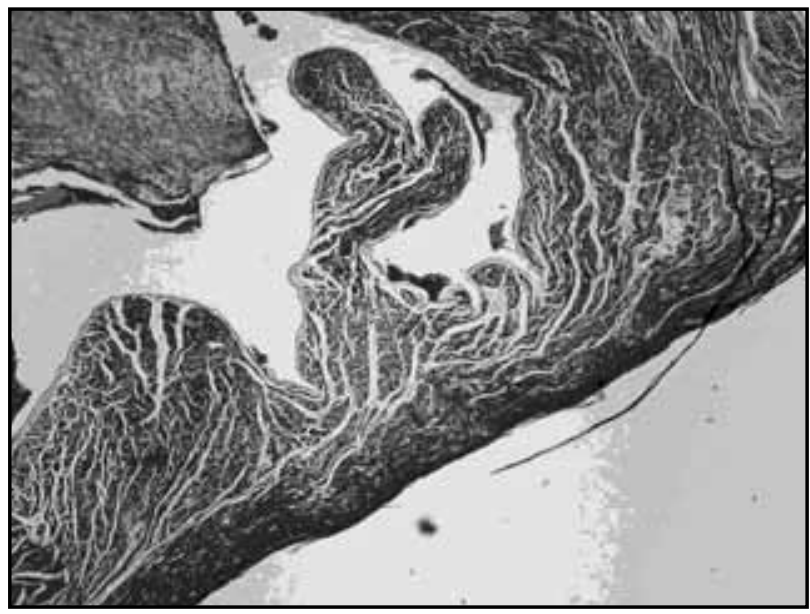

Figura 2 - Microscopia mostrando a não compactação do miocárdio

A história natural da doença não está bem estabelecida. Muitos pacientes apresentam sintomas mínimos ou não têm sintomas, e a progressão da doença nesses pacientes não é bem clara, embora haja evidências de que o prognóstico em longo prazo não seja bom ${ }^{(2,3,6,8)}$. Em pacientes sintomáticos, o curso da doença geralmente é aquele de deterioração progressiva da função cardíaca. Geralmente a doença tem início insidioso, com certa hipertrofia ventricular compensatória e dilatação ventricular esquerda assintomática. Comumente, a intolerância aos exercícios físicos progride até insuficiência cardíaca congestiva (ICC) franca. Embora a causa da depressão progressiva da função do ventrículo esquerdo não esteja bem definida, os achados clínicos e ecocardiográficos lembram os encontrados na cardiomiopatia dilatada, com exceção de que, no miocárdio não compactado isolado, a parede do ventrículo esquerdo se encontra espessada nos segmentos apical e inferior. Assim, a reduzida contratilidade do miocárdio resulta em insuficiência cardíaca com grande volume diastólico residual, dilatação do anel valvular e insuficiência relativa das valvas mitral e tricúspide. Em decorrência da estase de sangue nas câmaras, pode haver formação de trombos locais ao nível dos profundos recessos intertrabeculares com complicações pulmonares ou sistêmicas por tromboembolismo.

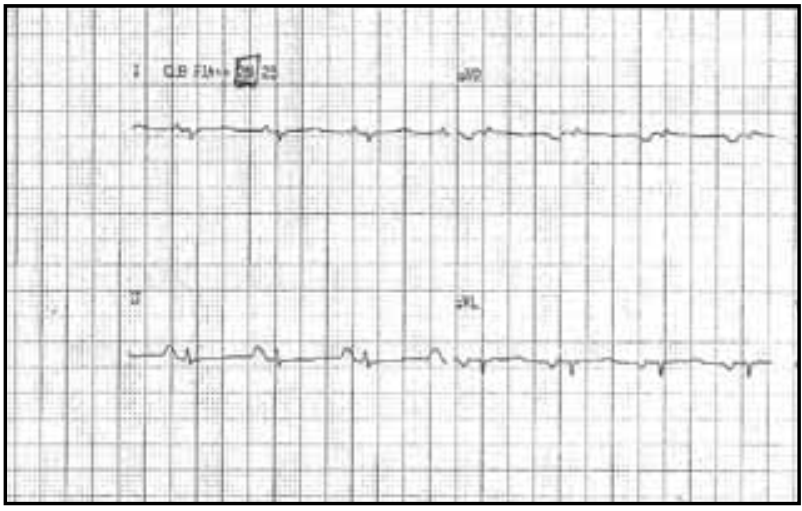

Figura 3 - Eletrocardiograma mostrando baixo potencial difuso com alteração da repolarização ventricular

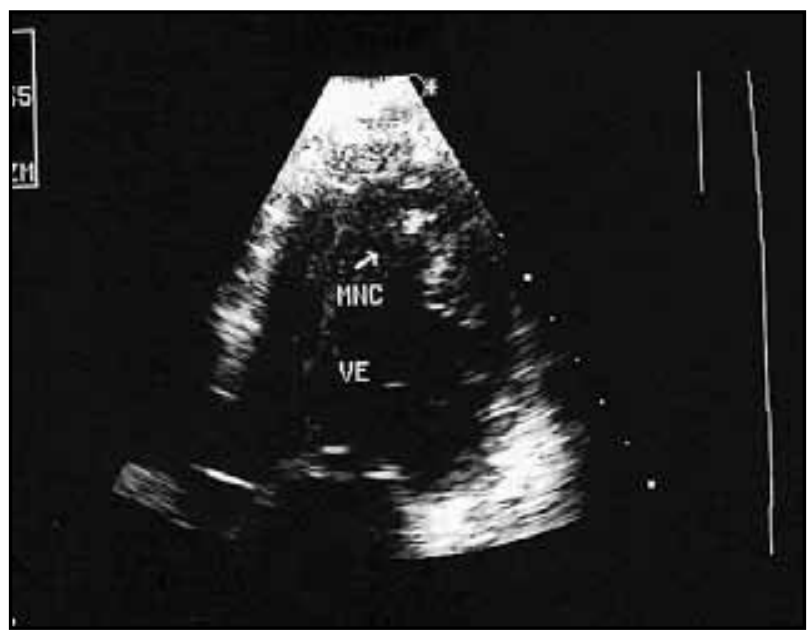

Figura 4 - Ecocardiograma mostrando área de não compactação miocárdica no ventrículo esquerdo

\section{Conclusão}

Os achados morfológicos característicos do miocárdio não compactado podem ter comprovação diagnóstica ao ecocardiograma bidimensional com Doppler, e correspondem aos achados morfológicos macroscópicos durante a necropsia. Seu diagnóstico deve ser considerado nos pacientes com achado de falência ventricular, arritmia ventricular e eventos embólicos.

Para realizarmos diagnósticos precoces, necessitamos do conhecimento dessa patologia por parte dos profissionais que estarão envolvidos com o início da vida dos pacientes, como geneticistas e pediatras, podendo dessa maneira tomar as medidas necessárias, a fim de evitar ao máximo suas consequências.

O tratamento clínico dos pacientes com disfunção ventricular seria o habitualmente utilizado nos pacien- 
tes com falência ventricular, devendo-se considerar o transplante cardíaco como opção nos casos refratários à terapêutica mais conservadora. As incidências de falência ventricular, arritmia ventricular e embolizações devem chamar atenção para essa entidade rara, que é complicada por uma considerável morbidade e alta mortalidade.
Além disso, devido à associação familiar descrita(2, 5, 6), torna-se necessário realizar estudo ecocardiográfico nos pacientes. Apesar do alto índice de mortalidade, o presente relato sugere que casos com boa função miocárdica e ausência de arritmias demonstrada podem apresentar bom prognóstico.

\section{Referências}

1. BORREGUERO, L. J. et al. Images in cardiovascular medicine. Diagnosis of isolated noncompaction of the myocardium by magnetic resonance imaging. Circulation, v. 105, n. 21, E177-8, 2002.

2. CHIN, T. K. et al. Isolated noncompaction of left ventricular myocardium. A study of eight cases. Circulation, v. 82, n. 2, p. 507-13, 1990.

3. ESPINOLA-ZAVALETA, N. et al. Non-compacted cardiomyopathy: clinical-echocardiographic study. Cardiovasc Ultrasound, v. 4, p. 35, 2006.

4. HAMAMICHI, Y. et al. Isolated noncompaction of the ventricular myocardium: ultrafast computed tomography and magnetic resonance imaging. Int $\mathrm{J}$ Cardiovasc Imaging, v. 17, n. 4, p. 305-14, 2001.

5. ICHIDA, F. et al. Clinical features of isolated noncompaction of the ventricular myocardium: long-term clinical course, hemodynamic properties, and genetic background. J Am Coll Cardiol, v. 34, n. 1, p. 233-40, 1999.

6. MARON, B. J. et al; American Heart Association; Council on Clinical Cardiology, Heart Failure and Transplantation Committee; Quality of Care and
Outcomes Research and Functional Genomics and Translational Biology Interdisciplinary Working Groups; Council on Epidemiology and Prevention.Contemporary definitions and classification of the cardiomyopathies: an American Heart Association Scientific Statement from the Council on Clinical Cardiology, Heart Failure and Transplantation Committee; Quality of Care and Outcomes Research and Functional Genomics and Translational Biology Interdisciplinary Working Groups; and Council on Epidemiology and Prevention. Circulation, v. 113, n. 14, p. 1807-16, 2006.

7. OECHSLIN, E. N. et al. Long-term follow-up of 34 adults with isolated left ventricular noncompaction: a distinct cardiomyopathy with poor prognosis. J Am Coll Cardiol, v. 36, n. 2, p. 493-500, 2000.

8. RITTER, M. et al. Isolated noncompaction of the myocardium in adults. Mayo Clin Proc, v. 72, n. 1, p. 26-31.

9. TAMBORINI, G. et al. Incidence and characteristics of left ventricular false tendons and trabeculations in the normal and pathologic heart by second harmonic echocardiography. J Am Soc Echocardiogr, v. 17, n. 4, p. 367-74, 2004. 